\title{
LA ESCUELA EN LA ENCRUCIJADA DEL CAMBIO EPOCAL
}

\author{
Guillermina Tiramonti*
}

\begin{abstract}
RESUMEN: La escuela es una producción institucional de otro momento histórico y que, por lo tanto, nació asociada a otras circunstancias sociales, políticas y culturales. El artículo presenta y analiza aquellos ítems que la autora considera de peso para mesurar las asimetrías existentes entre la institución escolar y el momento histórico y cultural en el que ésta se inserta. Para ello se analizan los cambios en el entramado institucional construido a partir de la sociedad industrial haciendo especial referencia a las transformaciones en la familia, el Estado y la propia escuela. Se discute el contexto cultural contemporáneo y las distancias existentes entre este y la cultura letrada que moldeo la escuela moderna. Por último se plantea que la escuela es una institución que se pensó en base a una secuencia temporal asociada a la idea de progreso social e individual que privilegia la construcción para el futuro y hoy esta sometida a una demanda de generar un presente gratificante para los jóvenes.
\end{abstract}

Palabras claves: Jóvenes. Educación. Cambio cultural. Cambios institucionales. Escuela media.

\section{SCHOOL IN TIMES OF EPOCHAL CHANGE}

ABSTRACT: Associated to other social, political and cultural circumstances, school was institutionally created in a different historical moment. This paper presents and analyzes items that allow us to measure the existing asymmetry between school as an institution and the historical and cultural time in which it is nowadays inserted. The changes in the institutional network brought by the industrial society are thus analyzed, especially those related to the family, the state and school itself. The contemporaneous cultural context and the gaps between it and the literate culture that shaped modern

* Directora de la Facultad Latinoamericana de Ciencias Sociales (FLACSO, Argentina). E-mail: tiramonti@flacso.org.ar

Educ. Soc., Campinas, vol. 26, n. 92, p. 889-910, Especial - Out. 2005 
school are discussed. Our argument is that although school as an institution was thought on the basis of a temporal sequence linked to the idea of individual and social progress, which privileges a construction for the future, it is currently required to generate a gratifying present to young students.

Key words: Young people. Education. Cultural change. Institutional changes. High school.

D

espués de una década y media de reformas educativas que se pretendían bendecidas por el poder de la verdad y el conocimiento científico, la educación sigue siendo una cuestión no resuelta y últimamente escasamente ventilada en la esfera de la discusión pública. Hay en general una tendencia a pensar sus problemas como la derivación de un funcionamiento deficitario de la propia institución, de sus agentes o de las comunidades a las que atienden. Se pone el acento en una suma de déficit puntuales que si se atendieran adecuadamente a través de más presupuesto, disciplinamiento de los agentes y la debida preocupación de los padres, las cosas mejorarían sustancialmente. Sin embargo, la problemática que enfrenta la escuela tiene otra envergadura y un grado de complejidad que exige una mirada más amplia y abarcativa de cambios epocales que se articulan de un modo particular con la institución escolar.

Lo primero a decir es que la escuela es una producción institucional de otro momento histórico y que, por lo tanto, nació asociada a otras circunstancias sociales, políticas y culturales.

Nos proponemos en este artículo presentar y analizar aquellos ítems que consideramos de peso para mesurar las asimetrías existentes entre la institución escolar y el momento histórico y cultural en el que ésta se inserta.

\section{Los cambios en el entramado institucional}

La escuela moderna es una construcción social surgida entre el siglo XVII y XVIII en las sociedades europeas que inicia una inédita relación que denominamos pedagógica entre un maestro (en un sentido nuevo del término) y un alumno. La relación es inédita porque el maestro no es más un artesano transmitiendo un saber-hacer a un joven aprendiz, sino que se autonomiza del resto de las relaciones sociales y genera 
un espacio y tiempo especifico para la transmisión de los conocimientos (Vincent, Lahir y Thin, 2001).

El espacio escolar organiza las actividades de enseñanza aprendizaje mediante su distribución del tiempo y el espacio. La graduación de los alumnos para su distribución en aulas y la división del tiempo que marca la duración de la jornada diaria, el período del año en el que se desenvuelven las actividades y el lapso de la vida que se ocupa en ella.

La hipótesis de Ariès acerca de la construcción de la infancia es una de las principales referencias de los estudios socio históricos en la materia. El autor señala que dicho proceso tuvo lugar en las sociedades europeas a partir del siglo XVII, cuando comenzaron a delinearse nuevos sentimientos y afectos respecto a la niñez, opuestos a una mentalidad que los pensaba como adultos pequeños. La aparición del cuerpo infantil implicó una serie de transformaciones relacionadas con un lento proceso de demarcación de la niñez, la percepción del niño en tanto ser inacabado que necesitaba resguardo y su segregación y posterior reinserción en la sociedad. En este proceso, la escuela fue causa y consecuencia, relacionada principalmente con la necesidad de alejar al niño de la vida cotidiana del adulto. Asimismo, implicó una proliferación de discursos para la regulación del cuerpo infantil provenientes de la psicología, la pediatría, la pedagogía y posteriormente el psicoanálisis.

Toda aparición de una determinada forma social está ligada a otras transformaciones que se suceden en el mismo momento histórico, que la constituyen como piezas de un mismo entramado societal y le otorgan un determinado sentido político. La conformación de los Estados nacionales asociado a las exigencias del gobierno de una población definida como libre es sin duda uno de los referentes a considerar cuando se sitúa el surgimiento de la escuela moderna y se intenta reconstruir el sentido político de esta creación institucional. Forman parte del mismo paquete histórico los procesos de secularización del orden social, la conformación de lo que ha dado en llamarse la familia burguesa y finalmente el desarrollo del capitalismo industrial.

Por supuesto este entramado adquiere diferentes características en los distintos países, ya sea que hagamos la distinción entre los europeos y latinoamericanos, o que diferenciemos situaciones específicas en América Latina. En este sentido es necesario considerar las distancias existentes entre los modos de incorporación y articulación societal que caracte- 
rizan a países como Brasil y Argentina. En el primer casó se trata de una conformación que deja fuera del alcance del Estado una parte considerable de la población, constituyendo una sociedad dual que el crecimiento económico y social no ha podido neutralizar. En el otro caso, el argentino, se trata de un modelo que se propone la integración y articulación del conjunto de la sociedad a partir de un proceso de fuerte homogeneización cultural realizado por el Estado a través de la educación pública.

De cualquier modo el modelo societario con que se organizaron las sociedades latinoamericanas desde fines del siglo XIX hasta avanzada la segunda mitad del siglo XX tuvo al Estado como protagonista. Formando parte de esta matriz societal, que Marcelo Cavarozzi (1999) denominó estado céntrica, se constituyó la escuela pública en América Latina. El sistema que se constituye a fines del siglo XIX y principios del $\mathrm{XX}$ reconoce en el Estado nacional su principal referente material y simbólico tanto para la administración, la gestión y el financiamiento de las instituciones escolares como para la provisión de un sentido que se pretendía universalista y que expresaba en clave nacional la "cultura civilizada" (Tiramonti, 2003).

En este modelo societal, el Estado es una figura clave en la construcción de un espacio de sentido para el conjunto de los individuos y de las instituciones. La escuela estuvo doblemente asociada a la creación de este espacio común: por una parte como portadora de una propuesta universalista que expresaba el conjunto de los valores, los principios y las creencias en los que se fundamentaba la "comunidad", a la que debían incorporarse las nuevas generaciones y, por otra, como dispositivo de regulación social, y, en consecuencia, como instrumento de gobernabilidad.

Trabajos recientes (Romero, 2004) muestran cómo a través de los textos escolares se forjaron las representaciones de lo que éramos como nación, del pasado que compartíamos, de las tradiciones en las que se inscribía tanto el presente como nuestro destino como nación. La constitución de nuestras representaciones identitarias como sociedad, como comunidad de pertenencia, fue plasmada en el espacio escolar. Por supuesto buena parte de los sentimientos xenófobos provienen de esta demarcación de la ciudadanía nacional y su diferenciación con lo extranjero. Un croquis de una Argentina que es pura frontera que se marca con un trazo muy fuerte transmite toda una concepción de las distancias entre nosotros y los extranjeros. 
Al decir de Antonio Bolívar (2004), la institución escolar, que en su origen desplegó un ejercicio que consistía en subordinar las identidades históricas y culturales particulares al proyecto de creación de una identidad nacional, esta hoy involucrada en responder a la multiculturalidad.

La formación de la nacionalidad se correspondió con la conformación de la sociedad industrial y con el entramado institucional propio de esta etapa del desarrollo capitalista: la fábrica, la familia, la escuela, la clase social y las instituciones de representación política y sectorial constituyeron marcos institucionales que regulaban y contenían la existencia de los individuos hasta avanzado el siglo XX. Se trata de un complejo institucional cuya eficacia resultaba de la complementariedad de su acción y su común referencia al Estado nacional.

El proceso de globalización rompe esta matriz societal y deshace el entramado institucional en el que se sostenía y, con ello, el campo común al que se integran y articulan individuos e instituciones. El Estado, y por ende la acción política y los criterios que esta definía para la organización del campo nacional, pierde centralidad, en favor de una presencia fuerte del mercado y la competencia en la definición del orden societal. Son numerosos los posicionamientos respecto del nuevo lugar del Estado. Para algunos autores se trata de un estrechamiento de los grados de autonomía de la política y de la capacidad del Estado de controlar el conjunto de variables que impactan el campo nacional (Held, 1997), como resultado de la primacía de las corporaciones multinacionales que prefieren un mundo con Estados disminuidos o sin Estados.

Panitch (2000) introduce la temática de la asimetría en el poder de los Estados que interactúan en el orden globalizado y desarrolla la tesis acerca de que los Estados más poderosos han desempeñado un papel activo, y con frecuencia crucial, en el advenimiento de la globalización y en la tarea de mantener este nuevo orden. En posiciones más extremas, hay autores que plantean el arrasamiento de los modelos integrados de la posguerra. Touraine (1999) postula que lo que se llama globalización no es otra cosa que el retorno al capitalismo, entendiendo por ello la economía de mercado que rechaza cualquier control exterior y trata de usar todos los elementos de la sociedad como recursos adaptados a su propia finalidad. Más allá de las diferencias, hay coincidencias respecto de la ruptura de un campo social articulado e integrado por la acción del Estado. 
El nuevo tipo societario está acompañado por una serie de cambios en las instituciones que estructuraban la sociedad industrial y enmarcaban la existencia de los sujetos. Para Beck (1997), asistimos a una descomposición de la sociedad industrial como entramado de experiencias. Según esta postura, las personas han quedado libres de las seguridades y de las formas de vida estandarizadas. Así como la constitución de la sociedad industrial implicó el desencaje (Giddens, 1994) de las relaciones sociales del contexto local de interacción y las incorporó a un nuevo entramado relacional en el que el sexo, la familia y la clase definían un modo de vida y por lo tanto las condiciones de existencia, la sociedad postindustrial provoca la descomposición de ese entramado de experiencias desregulando la existencia de los individuos que están condenados a ser ellos mismos (Sennett, 1978). Se trata de la pérdida del sistema de referencias que proporcionaba la sociedad industrial y de la constitución de un sujeto autoreferencial.

Los cambios en este sentido son tan importantes que hay autores que hablan de la "declinación de la idea de sociedad" o de la muerte de lo social (Dubet y Martuccelli, 1998). Según este razonamiento, las instituciones habrían perdido la capacidad de marcar las subjetividades y estamos asistiendo al paso de una sociedad que integraba mediante un proceso de súper socialización de los agentes a través de diferentes agencias socializadoras, entre las cuales se destacan la familia, la escuela y la iglesia, a una sociedad de individuos subsocializados y anómicos. Se trataría de un proceso de desinstitucionalización que acompaña la crisis de la sociedad como concepto y como realidad (Tenti Fanfani, 2002).

En el campo de la educación, estos posicionamientos señalan la pérdida de la potencialidad de la escuela para instituir identidades y asocian esa caída con la muerte del Estado nación y de la ley como instancia fundadora de la ciudadanía. La escuela "cayó", según estos discursos, como ilusión forjadora de un sujeto universal y no dispone de ninguna narrativa en la que anclar la constitución de lo social (Duschatzky, 2001).

Sin adscribir a posicionamientos tan radicales, es evidente que la red institucional que caracterizó a la sociedad moderna se está modificando y que esto cambia los marcos estructurantes de la acción y el terreno en que se mueven las instituciones escolares. Los datos de nuestra investigación arrojan evidencia respecto de la existencia de procesos de 
este orden que afectan de modo diferenciado a los sectores y subsectores sociales, como así también, respecto de la permanencia y la consiguiente combinación con procesos anteriores y, en definitiva, de la desorganización de los antiguos marcos estructurantes de la acción y de la dificultad para identificar las orientaciones y características del nuevo tipo societario.

Es evidente que el modelo societal integrado por la acción política de un Estado con capacidad de articular e incluir material y simbólicamente al conjunto de la población - y de construir de este modo un lazo comunicante entre todos los miembros de la sociedad, y un campo común en la que se inscribían desigualdades y diferencias - está roto. Es claro también que el conjunto de las instituciones que caracterizaron a la sociedad industrial está atravesando profundas modificaciones y que esto, a su vez, da cuenta de una sociedad que está reconfigurando sus dispositivos de control y reproducción social. La pregunta es ¿̨cuánto y de qué modo esto se hace presente en los agentes e instituciones escolares?

En esta nueva orden societal la familia ha cambiado. Ha dejado de ser una estructura jerárquica organizada alrededor de los mandatos de reproducción de la especie, de la producción material y de las exigencias del mandato patriarcal. Hoy día el eje organizador de la unidad familiar es la comunidad emocional, el cultivo de la intimidad y el reconocimiento de las individualidades. Giddens (2000) habla de una relación "pura" para nombrar relaciones basadas en la comunicación emocional, en las que las recompensas derivadas de la misma son la base primordial para su continuidad.

Este tipo familiar resulta de una larga lucha por la emancipación de la mujer, en la que jugó un papel central la separación entre sexualidad y reproducción que resultó del desarrollo de los dispositivos de anticoncepción; de la valorización del espacio íntimo como fuente de gratificación; de la penetración del discurso "psi” y también de la perplejidad de los adultos frente a los nuevas configuraciones culturales. Esta nueva familia es sin duda mas débil en la transmisión de mandatos, no porque la composición heterogénea que ahora reviste impida este mandato, o porque las relaciones emocionales y la democratización de la intimidad obligue a una horizontalidad reñida con la transmisión cultural, o porque el discurso "psi" no incluya mecanismos de regulación, sino simplemente porque las familias viven la experiencia del cambio cultural 
y de una sociedad de riesgo que exige lecturas particularizadas de la realidad y construcción individualizada del futuro. Algunas de estas familias se han adaptado a esta sociedad de riesgo y apuestan a la creatividad de sus hijos para el despliegue de estrategias y trayectorias innovadoras para abordar un futuro siempre cambiante.

Los datos de una investigación ${ }^{1}$ reciente en la que participo muestran justamente las diferencias familiares en las estrategias de transmisión cultural. Sólo las familias provenientes de los sectores más tradicionales de la población encauzan a sus hijos en la preservación de una tradición que hasta el momento los ha mantenido en una posición social de privilegio. Las elites más modernas apuestan a la construcción de individuos competitivos y las clases medias ilustradas construyen una estrategia que combina recursos culturales con reforzamiento de la creatividad individual. El resto de las familias están sumidas en la perplejidad que les genera la comprobación de la "inoportunidad" de la transferencia a sus hijos de las antiguas estrategias a través de las cuales ellos lograron ascender socialmente. La vulnerabilidad de sus actuales posicionamientos o el descenso social que al que han sido sometidos en los últimos años les indican que las nuevas generaciones deberán innovar para afrontar con éxito el futuro.

Numerosos autores han tematizádo este fenómeno de ruptura generacional que se produce en una sociedad proyectada hacia el futuro. Ya en la década del setenta Bell (1977) sostenía que el problema sociológico de la realidad de nuestro tiempo - en términos de ubicación social e identidad - se plantea porque los individuos han soltado viejas amarras, ya no siguen pautas heredadas. De aquí el paso de la familia o la clase a la generación como fuente estructurante de la acción. Del mismo modo, Giddens (1997) acuñó el concepto de "modernidad reflexiva" para designar una sociedad donde la tradición se repliega y los individuos se ven forzados a vivir de una manera más abierta y reflexiva.

Sin embargo la retracción de la tradición, y las rupturas en la trasmisión generacional de estrategias concretas para la acción que por supuesto traen incluidas una definición ética, no significa que se haya interrumpido la trasmisión de capitales culturales y sociales a los hijos a partir de los cuales estos rearman sus trayectorias a la luz de las oportunidades y restricciones que generan las nuevas condiciones de existencia. No hay repetición pero si transferencia de capitales que, como señala Kessler (2000), no siempre pueden ser actualizado en las nuevas condiciones sociales. 
Los cambios en la familia han sido leídos en clave conservadora (Propuesta Educativa, n. 26, 2003) o con un dejo de nostalgia por la pérdida del orden patriarcal. Según estos autores las familias han dejado de cumplir con su función de socialización primaria, ya no transmiten a sus hijos una determinada visión del mundo sino que los han abandonado a sus propias elecciones. A partir de ello se hace un reclamo a las familias para que reasuman su función de autoridad y de socialización primaria de sus hijos de modo de restituir la capacidad familiar de regular los comportamientos de sus hijos.

En este caso, como en el de la queja por la acción de los medios de comunicación que veremos en el próximo punto, no se trata de la muerte de la familia ni de la pérdida de todo mecanismo de regulación, sino de una nueva configuración donde las responsabilidades individuales tienen otra centralidad y donde la regulación está más asociada a los flujos y redes en las que se inscribe la vida cotidiana. De cualquier modo, resulta impensable reconciliar la antigua estructura patriarcal de la familia con las nuevas reivindicaciones de libertad individual y realización personal de hombres y mujeres.

Según Beck, lo que se vive como crisis o catástrofe consiste en que tenemos que entender y reconocer más libertades de las que "habían sido previstas en el libro ilustrado de la mentada y prometida, pero nunca vivida, democracia". Vivimos, según este autor, bajo las condiciones de una "democracia internalizada" para la cual mucho de los conceptos y recetas de la primera modernidad se han vuelto insuficientes (Beck, 1999).

Esta libertad internalizada de la que habla Beck genera una diversidad que es visualizada como peligrosa en la medida en que no puede ser procesada por la red de instituciones sociales y políticas existentes. Pareciera que el camino para conciliar las instituciones con mayores grados de diversidad requiere abandonar la satanización de los individuos y sus familias.

Más allá de los temores que puedan ocasionar los procesos de individualización y las prácticas "no estandarizadas" que estos procesos generan, es importante señalar que son muchas las investigaciones que dan cuenta de una ruptura en la transmisión cultural intergeneracional. El cambio cultural dificulta la comunicación entre las generaciones. La generación electrónica es portadora de códigos, valores y comportamientos 
que resultan ajenos o extraños para la generación que les precede. Esta brecha generacional se ve agravada por la incertidumbre que genera el futuro de una sociedad en permanente cambio.

Esta dificultad para la transmisión está también presente en las escuelas. Hay una secundarización de la tarea de "enseñar" que podría resultar de la confluencia de varios factores: la importancia otorgada a las funciones asistenciales que recortarían el tiempo dedicado a las tareas específicas; la difusión de las teorías constructivistas y la cultura "psi" (Varela, 1991) que desplazan al adulto/docente de la posición de transmisor para ocupar el lugar de facilitador o guía de los aprendizajes espontáneos de los niños, o la brecha cultural entre adultos y jóvenes, a la que nos referimos en los párrafos precedentes, que imposibilita la función de transmisión. El primero de estos factores exige una acción por fuera de la escuela que reponga una situación de mayor equidad social y despeje a la institución de esta tarea asistencial, el segundo propone una reposición pedagógica de la función de transmisión cultural a través de la legitimación de esta acción, el último pone a la institución en posición de cuestionar su patrón cultural de modo de transformarlo incorporando críticamente los nuevos formatos culturales y retomar desde este lugar su función de transmisora cultural.

Como veremos en el apartado siguiente, la escuela pareciera estar en un lugar de resistencia cultural y no de apertura e intercambio con la cultura contemporánea.

\section{De la Galaxia Guttemberg a la sociedad mediática}

En la segunda mitad del siglo XX se acumularon una serie de cambios que transformaron significativamente el orden social, político, económico y cultural a la vez que modificaron la vida cotidiana de la gente e impactaron significativamente en la constitución de las subjetividades y en la conformación de identidades

En el campo de la cultura el fenómeno más significativo es sin duda la revolución de las comunicaciones, tanto por el desarrollo de los massmedia como por el de las tecnologías electrónicas para la transmisión y almacenamiento de datos. Sin duda los medios de comunicación se han constituido en un ecosistema o ambiente donde se desenvuelve nuestra vida y donde se recrean y producen lenguajes, conocimientos valores y orientaciones sociales (Quevedo, 2003). 
Este hecho desafía a la institución escolar, tanto en su función de transmisora de conocimientos y saberes, como en su carácter de socializadora de niños y jóvenes. En el apartado anterior hemos presentado la existencia de un proceso de des-institucionalización entendido como la pérdida de eficacia regulatoria de las instituciones generadas por la sociedad industrial entre las que se destacan el Estado, la escuela y la familia.

La otra cara de la moneda la constituye la capacidad de los massmedia para definir modos de vida, gustos y conformar un arco valorativo que reordena y desmonta las anteriores formas de intermediación y autoridad que configuraban hasta no hace mucho el estatuto del poder social (Barbero, 2002).

Las visiones o perspectivas más negativas depositan en los massmedia, fundamentalmente en la TV, el origen o la causa de todos los males que aquejan a la sociedad. De allí que se piense a la TV como un dispositivo que esta en la base del aumento de la violencia, la pérdida de la autoridad en todos los ámbitos y la decadencia de la capacidad lectora de las nuevas generaciones. En todos estos casos la TV es representada como un aparato poderoso que contrasta con la pasividad con que los niños y jóvenes recepcionan los mensajes.

A partir de esta apreciación sobre el efecto de los medios se ha construido un discurso sobre la muerte de la infancia. Desde este punto de vista, se considera que los medios han eliminado las fronteras entre la infancia y la madurez y por lo tanto han debilitado la autoridad de los adultos (Buckingham, 2002). A quienes así argumentan les preocupa las consecuencias de este pasar de fronteras. Plantean que la salud de los niños exige que nos transformemos en vigilantes de la línea de separación entre niños y adultos tanto en la casa, como en la escuela o en el ámbito general de la cultura. Esta separación implica una exclusión de los niños del mundo de los adultos. La amenaza de los medios electrónicos radica justamente en que constituyen una de las fuentes principales de conocimiento de la vida adulta. Desde esta perspectiva el dilema fundamental son el acceso y el control.

Podría decirse que la infancia se ha definido de diferentes modos a través de la historia y que estas definiciones han ido cambiando el estatus social de la niñez y sus relaciones con los adultos. La modernidad es la que establece esta separación tajante entre el mundo de los adultos y el de la niñez. El formato de la escuela moderna rompe con las anteriores 
formas de transmisión cultural que se basaban en un aprender a hacer a través de las incorporación de los aprendices a la producción familiar. La escuela instaura un lugar específico donde sucede la relación pedagógica que es autónomo al resto de la vida social y productiva (Vincent y otros, 2001). La extensión de los años de estudio y la jornada escolar estableció nítidas fronteras entre la vida de niños y adultos que los medios han desdibujado.

Sucede que los medios representan un papel cada vez más importante en la definición de las experiencias culturales de los niños de hoy. El desafío para las familias y la escuela no es protegerlo de los medios vedándoles el acceso, sino por el contrario prepararlos para abordar la experiencia mediática.

Dentro de este paquete de calamidades que se le atribuye a la influencia de los medios, esté el declinar de la lectura o la muerte del libro. Según esta postura la seducción que ejercen los medios audiovisuales sobre los niños y jóvenes sería la que explica su alejamiento de la lectura.

Sin embargo, la actual crisis de la lectura pareciera estar más relacionada con la profunda reorganización que atraviesa el mundo de la escritura y los relatos y la consiguiente transformación de los modos de leer, es decir, con el desconcierto que entre los mas jóvenes produce la obstinación de seguir pensando la lectura únicamente como modo de relación con el libro y con la pluralidad y heterogeneidad de textos y escrituras que hoy circulan. No se trata entonces de la muerte de la lectura, sino de la perdida de su lugar central y hegemónico en el espacio cultural. En el siglo XXI, aprender a leer los textos audiovisuales y los hipertextos es condición indispensable para la incorporación de las nuevas generaciones a un intercambio cultural que permita la constitución activa de la ciudadanía.

Hubo un tiempo en que el acceso al saber pasaba cuasi exclusivamente por la lectura fonética. Hoy sin embargo hay una heterogeneidad de textos a través de los cuales es posible acceder al conocimiento. La escuela fue constituida dentro del universo que Marshal MacLuhan bautizó como la Galaxia Guttemberg, es decir, un mundo dominado por la lógica del libro cuya base es la estructura de la linealidad y el orden secuencial (Quevedo, 2003, op. cit.). La heterogeinización de los textos rompe esta linealidad y modifica los modos de acceder al saber que tienen las nuevas generaciones. 
Según Barbero (2002, op. cit.), la actual ruptura generacional remite a una experiencia que no cabe en la linealidad de la palabra impresa pues nacidos antes de la revolución electrónica la mayoría de nosotros no entiende lo que esto significa. Este no entender pareciera ser lo que está sucediendo en las escuelas.

La inmersión de esta generación en un ambiente cultural tan diferente al de los mayores es en parte una de las causales de la existencia de una brecha generacional que es pensada como ruptura en el sentido de pérdida de los intercambios y de la transmisión cultural de una generación a otra. Esta ruptura también esta en la base de las dificultades de la escuela para constituirse en transmisora cultural.

Para algunos autores la escuela se ha transformado en un lugar de enfrentamiento entre la cultura letrada y la audiovisual. Sin embargo yo no creo que esa lucha efectivamente se esté librando. A mi entender, nos encontramos ante instituciones escolares en las que la transmisión cultural es poco significativa o muy débil, de modo que no puede considerarse que desde allí se apunte a contrarrestar o competir con el sustrato cultural que proponen los massmedia. Esta falencia de la función básica de la escuela resulta de su incapacidad de reconocer los nuevos códigos culturales y de poner en juego los instrumentos que proporciona la cultura letrada para interactuar inteligentemente con los medios audiovisuales y electrónicos.

Solo si la escuela asume a los medios como dimensión estratégica de la cultura podrá interactuar con los nuevos campos de experiencia surgidos de la reorganización de los saberes, los flujos de información, las redes de intercambios creativos y con la hibridación de las ciencias y las artes (Barbero, 2002).

$\mathrm{Al}$ mismo tiempo la mediatización de lo público exige una escuela capaz de proporcionar a las nuevas generaciones las habilidades cognitivas necesarias para leer e interpretar los mensajes mediáticos que lo interpelan como ciudadano. El ejercicio actual de la ciudadanía exige descodificar críticamente estos mensajes para establecer relaciones de sentido entre ellos los intereses que están en juego, los propósitos subyacentes y los objetivos declarados. La escuela debería proporcionar a los niños y jóvenes un "filtro cognitivo" que los desplace del lugar de espectador pasivo y lo transforme en un lector inteligente de los mensajes que se le dirigen ya sea como consumidor a través de las propagandas o como receptor cultural. 
Se trata de un cambio radical del proyecto cultural de la escuela. En primer lugar se trata de reconocer a las nuevas tecnologías de comunicación como tecnologías de intelectuales o sea como estrategias de conocimiento y no como meros auxiliares de la tarea escolar. En segundo lugar se trata de incorporar los medios audiovisuales como objeto de estudio de la cultura cotidiana de los chicos, de la sociedad en que vivimos, de los acontecimientos que jalonan nuestra historia y de los múltiples modos de contarnos esa historia. El análisis de una telenovela puede decirnos mucho de la cultura popular, de los valores que la articulan, de los modos que se procesa el conflicto en nuestras sociedades, del lugar de la mujer, de las relaciones familiares, de las relaciones entre los diferentes grupos sociales, de los modos de concebir la pobreza y la riqueza y así al infinito.

\section{De la promesa del futuro a la exigencia del presente}

La escuela es una institución pensada en la intersección del pasado, el presente y el futuro. Es sabido que es a través de ella (cada vez menos exclusivamente de ella) que se transmite de generación en generación un acerbo cultural, una versión de la historia, una valoración de los rasgos identitarios de la nacionalidad, una cosmovisión del mundo que reconoce tradiciones heroicas, que anula otras y que conforma lo que Wallerstein (1996) llama la versión nacional del arbitrario cultural de la civilización occidental. En esta invención del pasado la escuela aporta a la construcción de las representaciones que los argentinos tenemos de nosotros mismos, de nuestros vecinos, de nuestros derechos, del lugar que ocupamos en el mundo, del que deberíamos ocupar. Es desde allí que se entiende el presente y se construye una hipótesis sobre el futuro posible.

La mediatización de la cultura ha corrido en parte a la escuela de este lugar de exclusivo portador de la versión oficial de nuestra historia y con ello de nuestra identidad y destino. Es posible que los textos televisivos hagan hoy un aporte más sustancioso que la escuela para las construcciones identitarias y que en estas la relación con el pasado sea más efímera y menos importante como fundador del presente. Con esto queremos señalar que el relato escolar se sostiene en una secuencia temporal donde el pasado es fundante del presente, lo explica y lo hace inteligible. En cambio el discurso televisivo se justifica en un mero presente. 
Por otra parte, y siempre considerando esta secuencia entre pasado, presente y futuro, el relato escolar estableció un sentido teleológico entre pasado, presente y futuro. Tanto en la construcción de las trayectorias colectivas, de pueblos, naciones o etnias, como en los destinos individuales está presente esta idea moderna que existía una secuencia lógica y racional entre las opciones del pasado, las situaciones presentes y los destinos futuros. Todo el desarrollo intelectual del siglo XX estuvo anclado en esta visión de los caminos del "progreso", donde el presente se diluye y legitima por sus raíces en el pasado y por sus promesas para el futuro.

La escuela es una institución tradicionalmente anclada en esta secuencia temporal. El pasado que debe ser configurado, inventado, transmitido por la institución para construir una representación que haga inteligible el presente y justifique la pretensión de futuro. Al mismo tiempo, la escuela contiene una promesa de futuro. La promesa de integración e inclusión a través de la incorporación al mercado laboral y a los códigos del intercambio social y la promesa de la autonomía individual mediante el despliegue de las potencialidades que portamos como individuos. Desde esta perspectiva el presente escolar es valorado a partir de su capacidad de transmitir una versión del pasado socialmente aceptada y de seleccionar las tradiciones que abonan ese pasado y por otro lado, el presente se justifica porque contiene una hipótesis de futuro, una promesa a alcanzar.

Así como la modernidad modifico la percepción del tiempo y del espacio, posibilitando primero la separación entre ambos, los cambios acumulados en los últimos cuarenta años han transformado esta percepción de la modernidad (Ortiz, 1996). Habitamos una cultura que en la secuencia temporal se privilegia el presente. Esta entronización del "hoy" o del instante va de la mano de la pérdida de la ilusión del progreso, el escepticismo sobre el futuro y el desplazamiento de la ética del trabajo a favor de la estética del consumo. La demanda por gratificación que se ancla en el deseo y la búsqueda de su satisfacción, ha desplazado en gran medida una ética del sacrificio que desplazaba la graficación para el futuro (Bauman, 2000).

Nuestros datos de investigación son claros en cuanto la valoración de la gratificación y la realización personal tanto para padres, como para los jóvenes de los diferentes estratos de las clases medias. Este hecho ge- 
nera para las escuelas una demanda que antes no estaba presente o por lo menos no tenía la relevancia con que se presenta hoy.

Los padres evalúan no solo ni principalmente la construcción de futuro que promete la escuela, sino qué presente le brinda a sus hijos, de este modo se puede cambiar de escuela a los hijos en función de un presente evaluado como insatisfactorio.

Las escuelas recogen también esta exigencia: generar propuestas extracurriculares, darles afecto, protegerlos del medio social en el que viven, evitar el delito, aparecen como una exigencia que obliga a repensar la gramática escolar para reconfigurar la ecuación del tiempo en el espacio escolar.

La demanda por "contención" que recibe la escuela se inscribe en el mismo registro de valoración del presente. Este mandato de contener atraviesa a todas las instituciones escolares. Sin embargo son múltiples los sentidos que a el se le atribuyen. Así, para los docentes de algunas de las escuelas que atienden a los sectores altos de la población que en nuestras investigaciones hemos denominado de elites, el contener esta relacionado con el afecto y la comprensión que requieren jóvenes que no encuentran en sus familias atención amorosa acorde con las necesidades de su edad. Para otros docentes de este mismo grupo socio-económico contener esta más relacionado con establecer un marco disciplinario que regule las conductas acordes con una formación tradicional. Para los padres de estos mismos sectores el contener tiene otro sentido y está relacionado con más tiempo en la escuela y la oferta de otras actividades extracurriculares para sus hijos, o en el segundo caso la atención de un adulto significativo que escuche y oriente a los jóvenes.

Del mismo modo para una parte de los padres de clase media, su demanda de "contención" esta relacionada con un trato agradable y afectuoso de sus hijos y un ambiente adecuado para el desarrollo de las amistades juveniles. Se trata posiblemente de grupos familiares organizados horizontalmente, penetrados por la cultura "psi" y que han optado por un modelo de regulación de los hijos centrado en el autocontrol y la asunción individual de las responsabilidades y los riesgos. La investigación mostró muchas asimetrías en un proceso de individualización de los jóvenes. Para un grupo de los sectores medios y altos, es claro que hay una ampliación de las opciones individuales y una mayor presencia de decisiones autónomas. Dentro de estos mismos sectores socio-económi- 
cos hay jóvenes cuyas opciones siguen estando fuertemente condicionadas por las pertenencias de clase, por el entorno familiar o por la propia institución escolar. En el extremo más bajo de la escala social se repite la ampliación de la autonomía individual, solo que en este caso es difícil hacer una asociación con mayores grados de libertad, sino que estaríamos frente a lo que Castel (1997) llama individualización negativa o Robles (1999) individuación, nociones con las que se quiere dar cuenta de la situación de una parte de la población que está compulsivamente sometida al proceso de "ser ellos mismos" (Sennet, 2000) sin recursos y sin red de protección social.

Es en los sectores más bajos de la población donde este mandato adquiere una significación con fuerte contenido reproductor. Para muchos de los docentes que atienden a estos grupos sociales, la "contención" pasa por brindar un espacio institucional de protección social para aquellos que habitan en territorios caracterizados por la desintegración. Contener es proteger momentáneamente a sus alumnos de la violencia que caracteriza al medio social en el que habitan. En este caso la escuela es pensada como un espacio de aguante o como una institución que acerca a estos chicos algo que se parezca al mundo de los integrados. En un texto anterior hemos llamado a estas instituciones "escuelas para resistir el derrumbe". Y dentro de este grupo distinguimos las instituciones religiosas cuya propuesta tiene un claro componente pastoral, que contienen una promesa de "protección tutelar" y que mantienen una pretensión civilizatoria de aquellas otras que se proponen proporcionar una asistencia material y pedagógica y brindar un ámbito de "comprensión" y de "convivencia entre pares" para aquellos que participan activamente de la cultura del margen (Tiramonti, 2004).

En estos casos la escuela se propone socializar fortaleciendo la autoestima de los jóvenes. Ziegler (2004) señala que en esta intención confluyen principios de la pedagogía tradicional ligados al desarrollo de las potencialidades de los sujetos con tendencias "new-age", que procuran el fortalecimiento y conquista del yo a través de la autoayuda.

En esta línea de fortalecimiento del yo se inscriben las teorías de la resiliencia de amplia difusión en el campo pedagógico. La resiliencia se propone entender como niños, adolescentes y adultos son capaces de sobrevivir y superar adversidades a pesar de vivir en condiciones de pobreza, violencia o desastres naturales (Infante, 2001). En el campo esco- 
lar se proponen favorecer la autoestima y la autonomía, estimular la capacidad de resolver problemas y de mantener el buen ánimo a pesar de las situaciones adversas, y crear un clima de optimismo y alegría. Luthar y otros (2000) definen a la resiliencia "como un proceso dinámico que tiene como resultado la adaptación positiva en contextos de adversidad". Se distinguen tres componentes esenciales para la promoción de resiliencia: 1) la noción de adversidad, trauma o riesgo, 2) la adaptación positiva o superación de la adversidad, 3) la dinámica entre mecanismos emocionales, cognitivos y socioculturales que influyen sobre el desarrollo humano.

De allí que se piense a la escuela como un espacio que puede desarrollar una dinámica destinada a la adaptación positiva de niños y jóvenes que habitan en contextos sociales y familiares adversos. Es una propuesta que deposita en los individuos la responsabilidad de superación de las situaciones adversas, se trata entonces de una versión extrema de las exigencias de individualización a los que son sometidas las nuevas generaciones. Si el contexto no puede cambiarse entonces la única posibilidad es que los individuos desarrollen una estrategia "ganadora" para superar la adversidad del medio. Los sujetos son pensados como "superhombres" capaces de neutralizar las limitaciones estructurales.

Del mismo modo hay un discurso que proviene fundamentalmente de los ámbitos oficiales que demanda esta función "contenedora" de la escuela, que se expresa en la pretensión de universalización de los diferentes niveles educativos que viene atado tanto a un discurso que señala el valor del conocimiento en la sociedad actual, como a una retórica que asocia contención escolar con control del riesgo social y que piensa a la escolarización como un dispositivo de seguridad urbana, que saca a los jóvenes de la calle y previene la potencialidad delictiva de un grupo social que no estudia ni trabaja. Si bien la investigación ha mostrado que la vida delictiva de estos jóvenes se mantiene en paralelo a su asistencia a la escuela o el trabajo (Duschatzky y Corea, 2000; Kessler, 2002), esta asociación de escuela y prevención del delito esta muy presente y construye otra acepción para la exigencia de contención.

Las experiencias de escuelas "urbanas" que amplían la jornada escolar se inscriben en esta preocupación por el control del riesgo social. Se argumenta a favor de la doble escolaridad de los "pobres" en base a su derecho a recibir una escolarización "igual" a la que se les proporciona a 
los chicos "ricos" que generalmente concurren a escuelas con este formato escolar. Sin embargo, todas las investigaciones que han indagado sobre las instituciones que albergan este sector social (Almeida, 2002; Pinçon y Pinçot-Charlot, 2002) coinciden en señalar que este modelo de institución totalizadora tiene un función de reproducción del privilegio a través de la construcción de un cerco institucional que garantiza una socialización entre "nos", que permite controlar el mercado de amistades y con ello la conservación y ampliación del capital social a la vez que preserva de influencias extrañas modos de vida y las cosmovisiones del mundo en que estos se sustentan. Dada esta comprobación de los efectos reproductores del privilegio que alberga el modelo de institución total, es pertinente preguntarse por sus potenciales efectos en su aplicación en una población marginal, que justamente debe eludir el cerco de la pobreza y la exclusión.

Creemos no forzar la interpretación si señalamos que "el contener" denota una percepción de falta o de déficit de contención, que a nuestro criterio se relaciona con la escasa presencia de organizaciones e instituciones en el entramado social capaces de albergar la existencia de los jóvenes. Mas allá de la valoración que sobre la escuela tienen los diferentes actores, es claro que esta se ha transformado casi en la única institución con capacidad de albergar a este grupo etario. La escuela se ha constituido en "el' lugar para estar de los jóvenes" y esto la transforma institucionalmente en un espacio en el cual el presente y los modos de habitarlo adquieren una relevancia que no tuvo con anterioridad.

\section{A modo de cierre}

El artículo que aquí presentamos se propone contribuir en algo con la tarea de reconceptualizar el mundo en el que hoy las escuelas deben desenvolver la tarea de educar. El gran tema de hoy es redefinir los dispositivos tecnológicos con los que se pretende incorporar a las nuevas generaciones al orden social y cultural. La escuela moderna presenta limitaciones muy fuertes para cumplir una tarea que le sigue siendo reclamada. Responder a la pregunta de ¿Cómo educar hoy? exige revisar el sustrato cultural de las escuelas, su organización institucional, sus articulaciones con el entramado social, su papel en la reproducción de las diferencias sociales y en la búsqueda de la igualdad. En definitiva se trata de abandonar las pretensiones de reposicionar el mandato educativo de la 
La escuela en la encrucijada del cambio epocal

modernidad y plantearnos un nuevo proyecto institucional para dar acogida a las generaciones venideras.

Recebido e aprovado em agosto de 2005.

\section{Nota}

1. FlaCso/Secretaría de Ciencia, Tecnología e Innovación Productiva. Ministerio de Educación de la Nación Argentina. Proyecto: La nueva configuración educativa en la argentina. Investigación en curso. Dirige Guillermina Tiramonti.

\section{Referencias bibliograficas}

ARIÈS, P. El niño y la vida familiar en el Antiguo Régimen. Madrid: Taurus, 1987.

BARBERO, J.M. La educación desde la comunicación. Buenos Aires: Norma, 2002.

BAUMAN, Z. Trabajo, consumismo y nuevos pobres. Buenos Aires: Gedisa, 2000.

BECK, U. Teoría de la sociedad del riesgo. In: Beriain, J. (Comp.). Las consecuencias perversas de la modernidad: modernidad contingencia y riesgo. Madrid: Amorrortu, 1997.

BECK, U. Hijos de la libertad. Buenos Aires: Fondo de Cultura Económica, 1999.

BELL, D. Las contradicciones culturales del capitalismo. Madrid: Alianza Universidad, 1977.

BOLÍVAR, A. Ciudadanía y escuela pública en el contexto de diversidad cultural. Revista Mexicana de Investigación Educativa, México, DF, n. 20, p. 15-38, enero/marzo 2004.

BUCKINGHAM, D. Crecer en la era de los medios electrónicos, tras la muerte de la infancia. Madrid: Fundación Paideia; Morata, 2002.

CARLI, S. Niñez, pedagogía y politica: transformaciones de los discursos acerca de la infancia en la historia de la educación argentina entre 
1880 y 1955. Buenos Aires: Miño y Dávila, 2003.

CASTEL, R. La metamorfosis de la cuestión social. México, DF: Paidos, 1997.

CAVAROZZI, M. El modelo latinoamericano: su crisis y la génesis de un espacio continental. In: Garretón, M. América Latina: un espacio cultural en el mundo globalizado. Bogotá: Convenio Andrés Bello, 1999.

DUBET, F.; MARTUCCELLI, D. ¿En qué sociedad vivimos? Buenos Aires: Losada, 1998.

DUSCHASTZKY, S. Todo lo sólido se desvanece en el aire. In: Duschatzky, S.; Birgin, A. ¿Dónde esta la escuela? Buenos Aires: FlacsoManantial, 2001.

\section{LA ESCUELA EN LA CONSTRUCCIÓN DE LA HEGEMONIA.} Revista Propuesta Educativa, Buenos Aires, v. 22, n. 26, jul. 2003.

GIDDENS, A. Consecuencias de la modernidad. Barcelona: Alianza, 1994.

GIDDENS, A. Vivir en una sociedad postradicional. In: BECK, U.; Giddens, A.; Lash, S. Modernización reflexiva: política, tradición y estética en el orden social moderno. Madrid: Alianza, 1997.

GIDDENS, A. Un mundo desbocado. Madrid: Taurus, 2000.

HELD, D. La democracia y el orden global: del Estado moderno al gobierno cosmopolita. Madrid: Paidós, 1997.

KESSLER, G. Redefinición de las identidades sociales en tiempos de cambio: una tipología para la experiencia del empobrecimiento. In: Svampa, M. (Comp.). Desde abajo. Buenos Aires: Biblos, 2000.

NARODOWSKI, M. Infancia y poder: la conformación de la pedagogía moderna. Buenos Aires: Aique, 1999.

ORTÍZ, R. Otro territorio: ensayos sobre el mundo contemporáneo. Buenos Aires: UNQ, 1996.

PANITCH, L. El nuevo Estado imperial. New Left Review, Madrid, n. 3, p.5-6, jul./ago. 2000.

QUEVEDO, L. A. La escuela frente a los jóvenes, los medios de comunicación y los consumos culturales en el siglo XXI. In: TENTI 
FANFANI, E. Educación media para todos: los desafíos de la democratización del acceso. Buenos Aires: Altamira, 2003.

ROBLES, F. Los sujetos y la cotidianeidad: elementos para una microsociología de lo contemporáneo. Concepción, Chile: Sociedad Hoy, 1999.

ROMERO, L. A. (Coord.). La Argentina en la escuela: la idea de nación en los textos escolares. Buenos Aires: Siglo XXI, 2004

SENNET, R. El declive del hombre público. Barcelona: Península, 1978.

SENNET, R. La corrosión del carácter: las consecuencias personales del trabajo en el nuevo capitalismo. Barcelona: Anagrama, 2000.

TENTI FANFANI, E. Voz socialización. In: Altamirano, C. (Dir.). Términos críticos de la sociología de la cultura. Buenos Aires: Paidós, 2002.

TIRAMONTI, G. Estado, educación y sociedad civil: una relación cambiante. In: Tenti Fanfani, E. (Comp.). Educación media para todos: los desafíos de la democratización del acceso. Buenos Aires: Altamira, 2003.

TOURAINE, A. Globalización, fragmentación y transformaciones culturales en el mundo actual. In: Garretón, M. (Comp.). América Latina: un espacio cultural en el mundo globalizado. Bogotá: Convenio Andrés Bello, 1999.

VARELA, J. Las pedagogías psicológicas. In: Álvarez Uria, F.; Varela, J. Arqueología de la escuela. Madrid: La Piqueta, 1991.

VICENT, G.; LAHIR, B.; THIN, D. Sobre a história e a teoria da forma escolar. Revista Educação, Belo Horizonte, n. 33, p.7-48, jun. 2001.

WALLERSTEIN, I. Después del liberalismo. Madrid: Siglo XXI, 1996. 